\title{
Attrition in a 30-year follow-up of a perinatal birth risk cohort: factors change with age
}

Background: Attrition is a major cause of potential bias in longitudinal studies and clinical trials. Attrition rate above $20 \%$ raises concern of the reliability of the results. Few studies have looked at the factors behind attrition in follow-ups spanning decades.

Methods: We analyzed attrition and associated factors of a 30-year follow-up cohort of subjects who were born with perinatal risks for neurodevelopmental disorders. Attrition rates were calculated at different stages of follow-up and differences between responders and nonresponders were tested. To find combinations of variables influencing attrition and investigate their relative importance at birth, 5, 9, 16 and 30 years of follow-up we used the random forest classification.

Results: Initial loss of potential participants was $13 \%$. Attrition was $16 \%$ at five, $24 \%$ at nine, $35 \%$ at 16 and $46 \%$ at 30 years. The only group difference that emerged between responders and non-responders was in socioeconomic status (SES). The variables identified by random forest classification analysis were classified into Birth related, Development related and SES related. Variables from all these categories contributed to attrition, but SES related variables were less important than birth and development associated variables. Classification accuracy ranged between 0.74 and 0.96 depending on the age. Discussion: Lower SES is linked to attrition in many studies. Our results point to the importance of the growth and development related factors in a longitudinal study. Parents' decisions to participate depend on the characteristics of the child. The same association was seen also, when the child, now grown up, decides upon participation at 30 years. In addition, birth related medical variables are associated with the attrition still at the age of 30 . Our results using a data mining approach suggest that attrition in longitudinal studies is influenced by complex interactions of a multitude of variables, which are not necessarily evident using other multivariate techniques. 
4 Jyrki Launes ${ }^{1}$ M.D., Ph.D., Laura Hokkanen ${ }^{1}$ Ph.D., Marja Laasonen ${ }^{1,2}$, Ph.D., 5 Annamari Tuulio-Henriksson ${ }^{1,3}$, Ph.D., Maarit Virta ${ }^{1}$ Ph.D., Jari Lipsanen ${ }^{1}$ 6 M.A., Pentti J. Tienari ${ }^{4}$, M.D., Ph.D. and Katarina Michelsson ${ }^{5}$ M.D., Ph.D.

$7 \quad{ }^{1}$ Faculty of Behavioral Sciences, Division of Cognitive and Neuropsychology, University of

8 Helsinki, P.O. Box 9, 00014 Helsinki, Finland.

$9{ }^{2}$ Helsinki University Central Hospital, Department of Phonetics, Haartmaninkatu 8, 00290

10 Helsinki, Finland

$11{ }^{3}$ Kela - The Social Insurance Institution of Finland

$12{ }^{4}$ Biomedicum, Research Programs Unit, Molecular Neurology, University of Helsinki; Helsinki

13 University Central Hospital, Department of Neurology, Haartmaninkatu 8, 00290 Helsinki,

14 Finland

$15{ }^{5}$ Lecturer of pediatrics, retired

16 Correspondence to Dr. Launes at jyrki.launes@helsinki.fi 


\section{Introduction}

18 Bias arises if the rate of subjects dropping out from a longitudinal study or clinical trial is not random, and the variables responsible for a subject to drop out from the study are correlated with variables that are used to evaluate outcome. Such bias is referred to as attrition bias. In epidemiology the term attrition is often used as a proxy for not retaining subjects who were initially included in a study. Thus, significant attrition may also occur without causing bias i.e. significant imbalance between study groups. However, other types of bias may be caused when subjects are lost to follow-up (LTFU) e.g. loss of statistical power when the groups get smaller. One of the dictionary definitions of attrition is to become weaker over the course of time and when used in conjunction with studies the term includes two sides of one problem i.e. the predictive power becomes weaker and the number of participants gets smaller. It is accepted that $5 \%$ of cases lost-to-follow-up is acceptable and attrition of up to 20 percent rarely presents serious bias (the $20 \%$ rule) (Fewtrell et al., 2008) In evidence based medicine LTFU of more than 20 percent downgrades a Level 1 study to a Level 2 study. The literature reports on a number of cohort studies and clinical trials, with attrition rates not disclosed. The $20 \%$ rule may be one reason for the authors' reluctance to reveal high attrition rates (Fewtrell et al., 2008). Simulation studies suggest that the proposed 60-80 \% threshold of follow-up may cause significant bias especially when attrition is not completely random (Kristman, Manno, \& Côté, 2004). In order to interpret follow-up results, bias must be analyzed, corrected, and taken into account in clinical interpretation (Guyatt, 2009). However, even high proportions of LTFU do not necessarily cause uncontrollable bias for endpoint measures of a trial (Lacey, Jordan, \& Croft, 2013)(Gustavson, von Soest, Karevold, \& Røysamb, 2012), e.g. a high proportion of LTFU makes it unreliable to calculate prevalences but cause - consequence analyses are still possible (Martikainen, Laaksonen, Piha, Lallukka, 2007). In follow-up studies and trials that last for decades, it is next to impossible to achieve attrition less than $20-40 \%$. Although bias is a major concern in the literature, surprisingly few attrition analyses have revealed substantial bias in estimated measures (Littman et al., 2010).

44

45

46

47

48

49

50

51

52

53

54

55

56

57

58

59

There are many characteristic features for individuals dropping out of studies, which are seen rather constantly: male gender, unmarried, smoking, low socioeconomic status (SES), and poor health (Goldberg, Chastang, Zins, Niedhammer, \& Leclerc, 2006)(Young, Powers, \& Bell, 2006). Studies with aged subjects show a tendency of non-responders being older (Chatfield, Brayne, \& Matthews, 2005), but in studies with younger adults the opposite may be the case (Deeg, 2002) (Young et al., 2006). Other sociodemographic factors linked to attrition include fewer years of education, ethnic group, no previous participation in research, and major depressive disorder (Lamers et al., 2012)(Fröjd, Kaltiala-Heino, \& Marttunen, 2011). Finally, although the effect of personality of the subjects on attrition has not been extensively studied, personality, health, and lifestyle seem relatively neutral with respect to non-response (Distel et al., 2007).

Sometimes studies do not achieve good participation, even though previous experiences suggest otherwise (Korkeila et al., 2001). In one such study (Korkeila et al., 2001), the socioeconomical and behavioral features were important for non-response, but issues with privacy may have been decisive. A steady decrease of willingness to take part in studies has been observed in the western world in recent decades (Galea \& Tracy, 2007). This has been attributed to profound changes of the day-to-day social and working environment, but especially 
60

61

62

63

64

65

66

67

68

69

70

71

72

73

74

75

76

77

78

79

80

81

82

83

84

85

86

87

88

89

90

91

92

93

94

95

96

97

98

99

100

101

102 to advances in communication technology that cause an increase in unsolicited contacts in form of aggressive telemarketing and campaigns cloaking as research studies. All these practices are thought to cause general mistrust to scientific studies in general, and cause different likelihood of participation, depending on the nature of the phenomenon studied. A potentially harmful environmental factor e.g. toxic chemicals to which a subject has been exposed may increase the probability of participating a study of that factor, but a potentially embarrassing life style habit (such as alcohol abuse or smoking) may discourage participation in a study of that habit.

Numerous practices have been suggested to overcome difficulties of attrition and enrolment. These include repeated mailings, telephone contacts, hybrid enquiries with the options of responding by either postal or internet response, and financial compensation. Face to face contact is associated with better participation rates in health promotion programs (Park et al., 2011). Perceived benefit of being involved counterbalances the degree of inconvenience caused by participating.

In studies involving children, the balance between wanting to participate and finding it inconvenient becomes complex, as both the parents' and child's motivation affect participation. This relationship also changes at different age levels. Intuitively it would seem natural that parents of sick children are motivated to participate because of more individualized follow-up and treatment. However, a meta-analysis of trials of children with chronic medical conditions, such as asthma, obesity, arthritis, diabetes, cancer, sickle cell disease, and cystic fibrosis showed a mean rate of enrollment refusal as high as 37\% (range 0-75\%), the initial mean followup attrition of $20 \%$ (range $0-54 \%$ ), and extended follow-up attrition of $32 \%$ (range $0-59 \%$ ) (Karlson \& Rapoff, 2009). On the other hand, in the 26 year follow-up prospective cohort study in the United Kingdom on 1064 children born small for gestational age, the participation on followup visits at $5,10,16$, and 26 years was $93 \%, 80 \%, 72 \%$, and $53 \%$, respectively (Strauss, 2000). Up to the age of 10, the parents' decision whether to participate or not has likely been decisive, at 16 the adolescents have had probably started to express their opinion and at 26 years, the now adult offspring have decided independently on participation. It is unlikely that the same causes of attrition apply at all phases of such long follow-up cohorts. There is no direct data about parents' motives to respond, but in a 11-year longitudinal study of young females, frequent responders were likely to be more educated, less likely to be stressed about money, to smoke and to have children (Powers \& Loxton, 2010). The importance of the child's role can be evaluated from a study of parent-child interaction in families of preschoolers. Seventy-one percent of treatment dropouts were identified by lower SES, but poor child-parent-interaction was equally important (Fernandez \& Eyberg, 2009). However, in cohort studies lasting for decades, withdrawing from a cohort study may also be temporary (Goldberg et al., 2006).

Clinical, psychological, and social features that could be identified at study entry influence attrition among adults. However, studies involving children are more complex in recruitment and retention. We report here attrition in a 30-year follow-up cohort of subjects who were born with a predefined perinatal risk for neurodevelopmental disorder. The aim is to analyze the causes of attrition, as well as to identify changes in the importance of factors associating to attrition at different stages of the follow-up. We hypothesize that factors contributing to attrition change due to the change of the person deciding on participation. 


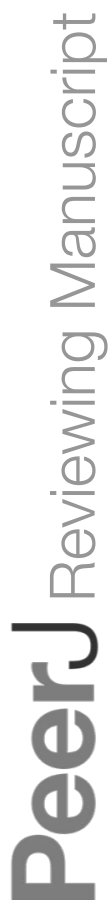

PeerJ reviewing PDF | (v2013:01:225:1:1:NEW 18 Jun 2014) 


\section{Base cohort}

105 The birth cohort originates from Kätilöopisto maternity hospital, Helsinki, Finland, in 1971 - 1974

106 and has been prospectively studied up to adulthood. The hospital is one of two major maternity

107 hospitals, the other being the University Central Hospital, in the Helsinki metropolitan area of

108 approximately 900.000 residents at the time of enrollment. The hospital served as the primary

109 maternity hospital serving an area of approximately $4000 \mathrm{~km}^{2}$. Sixty percent of the mothers lived

110 in Helsinki (mean distance to hospital 8 km), 7\% in Espoo (distance 15 km), 15\% in Vantaa

111 (distance $14 \mathrm{~km}$ ) and the remaining 15\% in the surrounding municipalities (average distance to

112 hospital $30 \mathrm{~km}$ ) (figure 1). All risk pregnancies known in advance as well as expected birth

113 complications, were directed to the University Central Hospital equipped with a neonatal

114 intensive care unit. Those born in Kätilöopisto therefore represent an unselected sample of

115 deliveries within the area.

116 There were 22,359 consecutive births, which at the time accounted for about $10 \%$ of all births in

117 Finland and about one third of those in the county of Uusimaa. Of those, 2113 (9.5\%) were

118 cesarean sections. Newborns were consecutively screened for predefined perinatal risks:

119 APGAR score lower than 7 at 5 or 15 minutes $(n=260)$, birth weight under 2000 grams or less

120 ( $n=221)$ out of whom 46 under 1500 grams, significant hyperbilirubinemia $(n=353)$, severe

121 respiratory difficulties including infections $(n=83)$, neurological symptoms $(n=114)$, maternal

122 diabetes ( $n=86)$, infant hypoglycemia ( $n=92)$. Altogether $1196(5.3 \%$ of the 22,359 births) had at

123 least one risk, $22 \%$ of them had more than one risk factor. Additionally, 130 cases were

124 screened, but did not fulfill the inclusion criteria. Of the 1196 infants, 202 died or were severely

125 disabled and excluded from the study. The inclusion of patients is given in figure 2

\section{Follow-up and other clinical visits}

127 There were several clinical visits after birth, and the children were seen at 6, 12, 18, and 24

128 months by the physicians involved in the longitudinal study. Additionally, almost all infants were

129 seen by a nurse at child-health centers as part of a public healthcare program, at 6, 10, 18, 24,

130 and 48 months (Vuorenkoski L Vuorenkoski L, Mladovsky P \& Mossialos E, 2008). Vaccinations

131 were given on these visits following a schedule adapted from the WHO recommendations, and

132 the vaccination program was continued in school health care. Therefore, the children and

133 parents had frequent face-to-face contacts with health care personnel in addition to study visits.

134 Moreover, clinical visits and psychological and logopedic test sessions took usually several

135 hours, which means that the time parents were in contact with the study personnel was

136 substantial. The parents did not receive financial compensation. The participants of the study

137 had normal access to the public health care system at all time s, as the study was not meant to

138 substitute public health services.

\section{Methods}

140 At birth detailed data was recorded about maternal risk factors (e.g. smoking, prescribed

141 medication and radiological examinations), family genetic traits, and medical data of delivery. 
142 At five years, a clinical evaluation, age-appropriate psychological tests, logopedic tests, and

143 questionnaires were used. The clinical evaluation included the Neurodevelopmental screen

144 NDS, (Bax \& Whitmore 1973) (Michelsson, Ylinen \& Donner, 1981). The psychological

145 evaluation included the Frostig Developmental Test of Visual Perception (Frostig, Lefever \&

146 Whittlesey, 1966), the Dubowizt screening test (Dubowitz, Leibowitz \& Goldberg, 1977), and the

147 Draw a Person Test (Goodenough, 1926). The logopedic evaluation included the Illinois Test of

148 Psycholinguistic Abilities (Kirk, McCarthy \& Kirk, 1968), a systematic evaluation of speech and

149 articulation, and a Name writing test. The parents filled out a structured questionnaire regarding

150 the health, development and behavior of the child. In addition, some kindergartens were

151 contacted. The parents were also interviewed.

152 At nine years a clinical evaluation, age-appropriate psychological tests, logopedic tests, and 153 questionnaires were again used. The clinical evaluation included the Test of Motor Impairment

154 (Stott, Moyes \& Henderson, 1972) and the assessment of neurological signs (Lindahl,

155 Michelsson, \& Donner, 1988) (Stockman et al., 1986). The psychological evaluation included the

156 Draw a Person Test (Goodenough, 1926) and the Wechsler Intelligence Scale for Children

157 (Wechsler, 1949). The logopedic evaluation included the Illinois Test of Psycholinguistic Abilities

158 (Kirk, McCarthy \& Kirk, 1968), the systematic evaluation of speech and articulation, the

159 Diagnostic Reading and Writing tests for Finnish school children (Ruoppila, Roman \& Vasti,

160 1968, 1969) and the Name Printing Test (Reimer, Eaves, Richards, \& Crichton, 1975). The

161 parents filled out a structured questionnaire regarding the health, development and behavior of

162 the child (supplemental material S1). Parents were asked of the usefulness of the study and

163 rehabilitation measures initiated based on the visit at 5 years of age. The teachers filled out a

164 structured questionnaire regarding school performance and behavior as well as personality of

165 the child (supplemental material S2). The parents were also interviewed face-to-face.

166 At 16 years questionnaires were used for the whole cohort. A clinical evaluation and

167 psychological tests, not analyzed in this paper, were conducted for a subsample of children. The

168 clinical evaluation included the Test of Motor Impairment (Stott, Moyes \& Henderson, 1972) and

169 the assessment of neurological disorder (Lindahl et al., 1988) (Stockman et al., 1986). The

170 psychological evaluation included subtests from the Wechsler Adult Intelligence Scale

171 (Wechsler, 1955). The questionnaires included early versions of the Child Behavior Check List

172 for parents and the Youth Self-Report (Achenbach \& Edelbrock, 1983, 1987). The parents also

173 filled out a structured evaluation of the child's behavior and personality (supplemental material

174 S3, S4, S5).

175 At 30 years, a questionnaire was used to explore educational and occupational outcome, health,

176 and life satisfaction (supplemental material S6). It included also the ADHD Current Symptoms

177 Scale as the ADHD Childhood Symptoms Scale with each of the 18 DSM-IV (American

178 Psychiatric Association, 1994) diagnostic symptom criteria scored from zero to three depending

179 on the severity of symptoms (Barkley \& Murphy, 1998).

180 The family's socioeconomic status (SES) was recorded at all levels. The social class variable

181 was defined as the best score on a 5-item scale based on father's occupation, and if

182 unavailable, mother's occupation was used. Psychosocial distress score was formed to include

183 poor housing conditions, divorces, relocations, alcohol abuse, unemployment, family conflicts,

184 domestic violence, imprisonment, severe diseases, or mental problems in family. 
186 Participation frequencies were calculated at birth, 5, 9, 16 and 30 years. The children, who were not seen on the 5-year visit or any other visit thereafter, were included only in the analysis of 5year results. First, we tested for significant group differences with the Mann-Whitney-U test with participation at different age levels as the grouping variable. Ordinal and categorical variables were cross-tabulated, the Fisher's exact test or the Pearson Chi-square test were used for significance testing and Cramér's $\mathrm{V}$ for association. Regression methods were not used, because in our follow-up cohort, neither the dependent nor the predictor variables belong to the same pool of variance as the person who decides of participation changes in course of the follow-up. Bonferroni correction was used to correct the $p$ values for the required alpha level of 0.05 due to multiple significance testing. Statistica 12 software was used for the calculations (Statsoft, 2013).

To identify clinically meaningful patterns and combinations of explanatory variables, we performed a classification tree analysis using the random forest algorithm (Breiman, 2001a). In a situation with a very large numbers of predictors which are likely to interact in an unknown fashion, classification tree analysis (e.g. Rokach, 2008) provides a flexible and robust way to find the most important predictors of attrition. The present data was collected at fixed time points. Therefore, classification tree analysis was used instead of survival analysis, which is often used to analyze study designs where time is measured as a continuous variable. The basic idea of classification tree analysis is simple. Prediction of a response or class $Y$ from inputs $X_{1}, X_{2}, \ldots, X_{p}$ is done by growing a binary tree. At each internal node in the tree, one applies a test to one of the inputs $X_{i}$. Depending on the outcome of the test, one goes to either the left or the right subbranch of the tree. Eventually one comes to a leaf node, where prediction is made. This prediction aggregates or averages all the training data points that reach that leaf. In contrast to this, predictors like linear or polynomial regression are global models, where a single predictive formula is supposed to hold over the entire data space. As noted previously, when the data has lots of features which interact in complicated, and possible nonlinear ways, assembling a single global model can be very difficult. Detailed presentation of classification tree algorithms can be found e.g. in Breiman et al. (1984) and in Kohavi el al. (2002).

214 Data mining techniques are useful in exploratory studies (Mendez, Buskirk, Lohr, \&Haag, 2008) when data cannot be fit into models using goodness-of-fit methods and statistically significantly differences are difficult to interpret. As compared to such classical data mining situations the sample size in the present study is small. Therefore the random forest algorithm (Breiman, 2001a) was, because it prevents overfitting the model which is problem in classical classification tree analysis (Breiman, 2001b). Model accuracy was optimized by testing predictive accuracy, which was in the range of $0.71-0.97$ at different age levels. The Gini index (Gini, 1909) was used as a measure for variable importance. Gini index is the weighted mean of the improvement that each variable causes for the splitting criterion (Friedman 2001). Maximal classification accuracy and the most important variables using Gini importance score are reported (Sandri \& Zuccolotto, 2006). Random forest classification was done using the Salford system Predictive Modeler software (Salford Systems, 2013 http://www.salford-systems.com). In all analysis Bootstrap aggregating was used for training algorithm (Breiman, 1996). Detailed nontechnical description of random forest algorithm can be found in Touw et all (2013). 
Ethical review has been conducted over the course of the longitudinal study, and the latest approval was obtained from the Ethical Review Board of the Helsinki and Uusimaa hospital district in May 2013 (number 147/13/3/00/2013).

232

233

234

235

236

237

238

239

240

241

242

243

244

245

246

247

248

249

250

251

252

253

254

255

256

257

258

259

260

261

262

263

264

265

266

267

\section{Results}

\section{Attrition}

There were 130 infants who were seen neither at the 5-year visit nor on any later visit. The initial loss during the first 5 years therefore was $13 \%$. However, 92 of them had been seen at an early clinical visit or child health centers during the first 40 months of life, thus only 38 were completely LTFU after birth. At 5 years, 839 children were assessed ( $84 \%$ of those alive and not severely disabled, $n=994)$, at the age of 9 years $756(76 \%)$, including 25 children not assessed at 5 years. At the age of 16 years 560 responded (65\% of those who participated at either 5 or 9 years, $n=864)$ and at the age of 30 years 469 responded (54\%). The attrition rate thus increased from $16 \%$ at 5 years to 46 at 30 years. There was data on $78(8 \%)$ children on one age level only, on $152(15 \%)$ children on two levels, on $288(29 \%)$ children on three levels, and on 343 (35\%) on all four age levels of the study. Participation of subjects on the different age levels is given in figure 3 and 4 . At the age of 9 years $54 \%$ of the parents regarded participation in the study useful, $26 \%$ were undetermined, $13 \%$ did not answer that question, and $7 \%$ did not find participation useful.

\section{Group comparisons of responders and non-responders}

Between the groups of children who were not brought to clinical evaluations at all $(n=130)$ and those who did not participate at five years, but participated later $(n=25)$, there was a statistically significant difference only in the father's social class (mean responders $2.2 \pm 0.9$ and $2.6 \pm 1.0$ in non-responders). The social class was the only variable that was significantly different between responders and non-responders at all other age levels too, a lower socioeconomic class being associated with non-responding.

A complete list of 63 variables tested for group differences is presented as (supplemental table S7). Notably, the type of perinatal risk had no apparent effect on the number of visits at any age level (Kruskal-Wallis test $\mathrm{H}=8.10 \mathrm{p}=0.32$ ). Also, maternal age, gestational age, birth weight, or Apgar scores or the distance from home to the hospital had no statistically significant differences on any age level.

\section{Classification analysis}

Classification accuracy of classifying cases into responders and non-responders, and five of the most important variables with their relative importance are given in table. A complete list of variable importance is available as a supplement table (S8). The variables that got a high importance score in the random forest model were grouped into three categories: 1 . a category characterizing the mother, infant and delivery (Birth related), 2. a category characterizing the neurodevelopmental factors and body mass index (Development related), and 3. a category characterizing the socioeconomic status, perceived security of the child and living conditions of the family (SES related) . 
268

269

270

271

272

273

274

275

276

277

278

279

280

281

282

283

284

285

286

287

288

289

290

291

292
For assessing the response of not participating at all, the average importance of the variables selected by the random forest model was 0.50 (range $1.0-0.16 ; 21$ variables) in the Birth related category and 0.39 (range $0.55-0.17$; six variables) in the SES related category. At five years the average importance was 0.39 (range $1.0-0.13 ; 24$ variables) in the Birth related category, and 0.27 (range $0.41-0.08$; seven variables) in the SES related category. At nine years the average importance was 0.28 (range $0.52-0.09 ; 20$ variables) in the Birth related category, 0.39 (range $0.85-0.07 ; 13$ variables) in the SES related category, and 0.81 (range $1.00-0.71$; five variables) in the Development related category. At 16 years of age the average importance of variables in the Birth related category was 0.19 (range $0.44-0.03 ; 11$ variables), 0.14 (range $0.56-0.03 ; 14$ variables) in the SES related category and 0.36 (range $1.00-0.23$; 12 variables) in the Development related category. At 30 years of age the corresponding figures were 0.31 (range $0.87-0.10 ; 11$ variables) for in Birth related category, 0.25 (range $0.79-0.06$; 17 variables) in the SES related category and 0.50 (range $1.00-0.39$; 13 variables) in the Development related category (fig. 5).

Within the Birth related category, at all age levels, maternal age, gestational age, birth weight, and Apgar scores were in the upper quartile of the variable importance scores.

Within the SES related category, up to the age of 16 , the most important variables the model chose for the classification into responders and non-responders reflected the family's occupation, housing, domestic disputes, and distance to hospital. At 30 years, the most important variables reflected more the individual's characteristics, e.g. subjective feeling of security, plans for future, parent's opinions regarding the child and to a lesser degree the socioeconomical features of the family in which the individual was raised up.

Within the Development related category, the Neurodevelopmental screen was the most important variable for classifying into responders and non-responders at 16 and 30 years and it was the fifth most important variable at nine years.

\section{Discussion}

294

The aim of the study was to analyze the causes of attrition, as well as to identify changes in the

295

296

297

298

299

300

301

302

303

304

305

306

307 importance of factors associating to attrition at different stages of a 30-year follow-up of a cohort born with perinatal risks. Initial loss of potential participants was 13\%. Attrition was $16 \%$ at five, $24 \%$ at nine, $35 \%$ at 16 , and $46 \%$ at 30 years. There were very few differences iln group comparisons of responders and non-responders, when traditional significance tests were used. The only finding was a lower socioeconomic class being associated with non-responding at all age levels. Using random forest model the variables were grouped into Birth related, Development related, and SES related categories. Birth related variables were important predictors of responding pattern during the first 5 years of the follow-up. The Development related variables became highly important at 9 years and appeared important at 30 years of age. The importance of SES related variables remained stable all through the follow-up but compared to the other categories, did not exceed their importance at any time point.

Measuring the relative importance of variables affecting attrition in very long lasting longitudinal studies, or even demonstrating which variables have any effect at all, is difficult using traditional 
stochastic models such as logistic regression. Logistic regression assumes that the conditional probabilities are a logistic function of the independent variables, which cannot be true when predictors may originate from either the parent or the child. Data mining techniques can be used instead when data cannot be fit into regular models (Mendez et al., 2008). In this analysis, as group level differences were negligible, we felt it unnecessary to correct for covariates using multivariate methods. On the other hand, using the random forest classifier, a very accurate (up to $96 \%$ ) classification of cases as responders and non-responders was achieved. The classification target variable was participation or nonparticipation at birth, 5, 9, 16, and 30 years in the random forest analysis. It seemed reasonable to assume that factors affecting the attrition may be "inherited" over age levels. Thus, variables from all previous levels were included in the classification analysis, e.g. data from the risks and events of the pregnancy and delivery as well as data from 5 years were used as predictors for classification at 9,16 , and 30 years. This allows for an estimation of both the quality and the quantity of the most important factors, as well as how they change over time (Breiman, 2001a)(Breiman, 2001b)(Genuer, Poggi, \& TuleauMalot (2010).

At the age of nine, 16 and 30 years, variables in all of the three categories, i.e. birth, SES and development related categories, influenced the classification. On all age levels after 5 years, the variables belonging to the development related category had the most influence (figure 5, table) in the random forest model. The variables in this category are scores of cognitive tests, parents', and teachers' assessments, behavior and motor skills, as well academic achievement, e.g. school grades. Such properties have not been reported as attrition factors in studies with younger subjects, but in the elderly, e.g. cognitive status is an important factor(Chatfield et al., 2005).

As commonly observed in studies (Fewtrell et al., 2008), the important SES related variables included area of residency, low social class, marital status, smoking and mother's working during pregnancy. However, there were no statistically significant group differences other than SES level. The distance to hospital was among the most important variables in the classification analysis up to the age of five, possibly because traveling with small children requires more time and attention. There appears to be a qualitative change in the most important SES related variables between 16 and 30 years, and the subjective benefit from participation was not among the important variables until the age of 30 .

At every age level the birth related variables, maternal age, gestational age, birth weight, and Apgar scores, were in the upper quartile of the variable importance scores. The medical events during pregnancy and in delivery had substantial influence in classifying between responders and non-responders on first clinical visits. The effect was still evident at 30 years, despite the fact that at this age the decision to respond was made by the subjects themselves instead of their parents. So far, it has not been reported that birth related factors are "inherited" as classifiers of response pattern to adult children. Repeatedly measured variables are intercorrelated by nature, but owing to the random forest algorithm's capability to handle missing data and correlated variables, and good resistance to overfitting, we think that this finding is not a mathematical artifact.

The magnitude to which the parents' attitudes are transferred to progeny by social inheritance of attitudes and negative dispositions towards volunteer activities and research (Park et al., 2011) cannot be analyzed in our material. On the other hand, the frequent face-to-face contacts may 
352 have improved inclusion and staying in the study. In addition to the study visits, our cohort had

353 frequent scheduled contacts with both the public health care system (programs of child health

354 centers, school health care). Such frequent contacts are generally considered a motivational

355 factor for parents. In Finland, there is also clear social pressure in keeping children in these

356 programs, which is exemplified by a high success rate of vaccination programs running

357 simultaneously (Peltola et al., 1994). We regard it highly probable, that these frequent visits have

358 reduced over-all attrition, as more than one half of the parents regarded participation in the study

359 as useful and only $7 \%$ not useful at all. However, as there were differences in the social class of

360 the families and SES variables were important throughout the follow-up, frequent face-to-face

361 contacts may not have the desired effect in reducing bias.

362 We conclude that attrition in our cohort is well within the same range as other lengthy studies;

363 especially the nonresponse rate in young adulthood is strikingly similar to the 26-year follow-up

364 of children born small for gestational age (Strauss, 2000). Based on other literature, many of the

365 longitudinal cohorts have not been badly influenced by attrition bias. We found very few

366 variables that were significantly different in group comparisons of responders and non-

367 responders. However, the lack of statistically significant differences does not prove the absence

368 of clinically relevant difference. Using a classification analysis, we found a number of variables

369 that very accurately predicted response or nonresponse. These variables could be classified into

370 three main dimensions describing events of pregnancy and birth, growth and neurodevelopment,

371 as well as socioeconomic status. By performing this analysis, we aimed to demonstrate a much

372 more complex network of dependencies that has been described in previous studies. The most

373 important difference between previously reported studies and our analysis is methodological.

374 Using traditional statistical tools, we found only one foreseeable difference, father's

375 occupationally defined social group. Therefore, we are confident, that future subgroup analyses

376 of our cohort will be reasonably unbiased as other cohorts resembling ours. Analyses based on

377 data mining techniques seem to reveal unexpected interactions, but our method must be

378 confirmed by similar analyses. 


\section{References}

380

381

382

383

384

385

386

387

388

389

390

391

392

393

394

395

396

397

398

399

400

401

402

403
Achenbach, T. M. \& Edelbrock, C. Manual for the Child Behavior Checklist and Revised Child Behavior Profile. (University of Vermont Department of Psychiatry: Burlington, VT, 1983).

Achenbach, T. M. \& Edelbrock, C. Manual for Youth Self-Report and Profile. (University of Vermont Department of Psychiatry: Burlington, VT, 1987).

Barkley, R. A. \& Murphy, K. R. Attention-Deficit Hyperactivity Disorder: A Clinical Workbook (2nd. ed). (The Guilford Press: New York, 1998).

Bax, M. \& Whitmore, K. Neurodevelopmental screening in the school-entrant medical examination. Lancet.2, 368-370 (1973).

Breiman, L., J. Friedman, and R. Olshen, and C. Stone (1984), Classification and Regression Trees, Pacific Grove: Wadsworth.

Breiman, L. (1996). Bagging predictors. Machine learning, 24(2), 123-140.Breiman, L. (2001). Random forests. Machine learning, 45(1), 5-32.

Breiman, L. (2001). Statistical modeling: The two cultures (with comments and a rejoinder by the author). Statistical Science, 16(3), 199-231.

Chatfield, M. D., Brayne, C. E. \& Matthews, F. E. (2005). A systematic literature review of attrition between waves in longitudinal studies in the elderly shows a consistent pattern of dropout between differing studies. Journal of clinical epidemiology, 58(1), 13-19.

Deeg, D. J. (2002). Attrition in longitudinal population studies: Does it affect the generalizability of the findings?: An introduction to the series. Journal of Clinical Epidemiology, 55(3), 213215.

Distel, M. A., Ligthart, L., Willemsen, G., Nyholt, D. R., Trull, T. J. \& Boomsma, D. I. (2007). Personality, health and lifestyle in a questionnaire family study: A comparison between highly cooperative and less cooperative families. Twin Research and Human Genetics, 10(02), 348353. 
404

405

406

407

408

409

410

411

412

413

414

415

416

417

418

419

420

421

422

423

424

425

426

427

428

429

430

Dubowitz, L. M. S., Leibowitz, D., \& Goldberg, C. A (1977) Clinical Screening Test for Assessment of Intellectual Development in Four and Five-year-old Children. Developmental Medicine \& Child Neurology, 19, 776-782

Fernandez, M. A. \& Eyberg, S. M. (2009). Predicting treatment and follow-up attrition in parentchild interaction therapy. Journal of Abnormal Child Psychology, 37(3), 431-441.

Fewtrell, M. S., Kennedy, K., Singhal, A., Martin, R. M., Ness, A., Hadders-Algra, M., Koletzko, B. Lucas, A. (2008). How much loss to follow-up is acceptable in long-term randomised trials and prospective studies? Archives of disease in childhood, 93(6), 458-461.

Frostig, M., Lefever, W., \& Whittlesey, J. R. B. (1966) Administration and Scoring Manual for the Marianne Frostig Test of Visual Peception. Consulting Psychologists Press: Palo Alto, California.

Fröjd, S. A., Kaltiala-Heino, R. \& Marttunen, M. J. (2011). Does problem behaviour affect attrition from a cohort study on adolescent mental health? The European Journal of Public Health, 21(3), 306-310.

Galea, S. \& Tracy, M. (2007). Participation rates in epidemiologic studies. Annals of epidemiology, 17(9), 643-653.

Genuer, R., Poggi, J.-M. \& Tuleau-Malot, C. (2010). Variable selection using random forests. Pattern Recognition Letters, 31(14), 2225-2236.

Gini, C. (1909). "Concentration and dependency ratios" (in Italian). English translation in Rivista di Politica Economica, 87 (1997), 769-789

Goldberg, M., Chastang, J. F., Zins, M., Niedhammer, I. \& Leclerc, A. (2006). Health problems were the strongest predictors of attrition during follow-up of the GAZEL cohort. Journal of clinical epidemiology, 59(11), 1213-1221.

Goodenough, F. (1926) Measurement of intelligence by drawings. World Company: New York.Gustavson, K., von Soest, T., Karevold, E. \& Røysamb, E. (2012). Attrition and generalizability in longitudinal studies: findings from a 15 -year population-based study and a Monte Carlo simulation study. BMC public health, 12(1), 918.

431 Guyatt, G. H. (2009). LOST to follow-up Information in Trials (LOST-IT): a protocol on the 432 potential impact. Trials, 10, 40. 
Hand, D.J., Mannila, H., and Smyth, P., (2001). Principles of data mining, MIT Press.

434 Karlson, C. W. \& Rapoff, M. A. (2009). Attrition in randomized controlled trials for pediatric 435 chronic conditions. Journal of pediatric psychology, 34(7), 782-793.

436 Kirk, S. A., McCarthy, J., \& Kirk, W. D. (1968) The Illinois Test of Psycholinguistic Abilities. 437 Revised edition. University of Illinois Press: Urbana, IL.

Kohavi, R., \& Quinlan, J. R. (2002). Data mining tasks and methods: Classification: decisiontree discovery. In Handbook of data mining and knowledge discovery (pp. 267-276).

Korkeila, K., Suominen, S., Ahvenainen, J., Ojanlatva, A., Rautava, P., Helenius, H. \& Koskenvuo, M. (2001). Non-response and related factors in a nation-wide health survey. European journal of epidemiology, 17(11), 991-999.

Kristman, V., Manno, M. \& Côté, P. (2004). Loss to follow-up in cohort studies: how much is too much? European journal of epidemiology, 19(8), 751-760.

Lacey, R. J., Jordan, K. P. \& Croft, P. R. (2013). Does Attrition during Follow-Up of a Population Cohort Study Inevitably Lead to Biased Estimates of Health Status? PloS one, 8(12), e83948.

447

448

449

450

451

452

453

454

455

456

457

458

459

460
Lamers, F., Hoogendoorn, A. W., Smit, J. H., van Dyck, R., Zitman, F. G., Nolen, W. A. \& Penninx, B. W. (2012). Sociodemographic and psychiatric determinants of attrition in the Netherlands Study of Depression and Anxiety (NESDA). Comprehensive psychiatry, 53(1), 63-70.

Lindahl, E., Michelsson, K. \& Donner, M. (1988). Prediction of early school-age problems by a preschool neurodevelopmental examination of children at risk neonatally. Developmental Medicine I\& Child Neurology, 30(6), 723-734.

Littman, A. J., Boyko, E. J., Jacobson, I. G., Horton, J., Gackstetter, G. D., Smith, B., Hooper, T., Wells, T. S., Amoroso, P. J., Smith, T. C.; Millennium Cohort Study. (2010). Assessing nonresponse bias at follow-up in a large prospective cohort of relatively young and mobile military service members. BMC medical research methodology, 10(1), 99.

Martikainen, P., Laaksonen, M., Piha, K., Lallukka, T. Does survey non-response bias tthe association between occupational social class and health? (2007) Scandinavian Journal of Public Health 32(2) 212-215 
461

462

463

464

465

466

467

468

469

470

471

472

473

474

475

476

477

478

479

480

481

482

483

484

485

486

Mendez, G., Buskirk, T. D., Lohr, S. \& Haag, S. (2008). Factors associated with persistence in science and engineering majors: An exploratory study using classification trees and random forests. Journal of Engineering Education, 97(1), 57-70.

Michelsson, K. Y. A. \& Donner, M. (1981). Neurodevelopmental screening at five years of children who were at risk neonatally. Developmental Medicine I\& Child Neurology, 23(5), 427-433.

Park, M., Yamazaki, Y., Yonekura, Y., Yukawa, K., Ishikawa, H., Kiuchi, T. \& Green, J. (2011). Predicting complete loss to follow-up after a health-education program: number of absences and face-to-face contact with a researcher. BMC medical research methodology, 11(1), 145.

Peltola, H., Heinonen, O. P., Valle, M., Paunio, M., Virtanen, M., Karanko, V. \& Cantell, K. (1994). The elimination of indigenous measles, mumps, and rubella from Finland by a 12-year, two-dose vaccination program. New England journal of medicine, 331(21), 1397-1402.

Powers, J. \& Loxton, D. (2010). The impact of attrition in an 11-year prospective longitudinal study of younger women. Annals of epidemiology, 20(4), 318-321.

Reimer, D. C., Eaves, L. C., Richards, R. \& Crichton, J. U. (1975). Name-printing as a Test of Developmental Maturity. Developmental Medicine I\& Child Neurology, 17(4), 486-492.

Rokach, L. (2008). Data mining with decision trees: theory and applications (Vol. 69). World scientific.

Ruoppila, I., Roman, K., \& Vasti, M. (1968) Diagnostic Reading Tests for Grades II and III in the elementary school. Institute of Educational Research Report No 41. University of Jyväskylä: Jyväskylä.

Ruoppila, I., Roman, K., \& Vasti, M. (1969) Diagnostic writing tests for grades II and III in the Elementary School. Institute of Educational Research Report No 50. University of Jyväskylä: Jyväskylä. classification and the forward search (pp. 263-270). 
489

490

491

492

493

494

495

496

497

498

499

500

501

502

503

504

505

Stokman, C. J. et al. Assessment Of Neurological "Soft Signs" In Adolescents: Reliability Studies. Dev Med Child Neurol. 28, 428-439 (1986).

Stott, D. H., Moyes, F. A., \& Henderson, S. A. (1972) A Test of Motor Impairment. Brook Educational: Guelph, Ontario.

Strauss, R. S. (2000). Adult functional outcome of those born small for gestational age: twentysix-year follow-up of the 1970 British birth cohort. Jama, 283(5), 625-632.

Salford Predictive Modeler Version 7.0. Computer Software. Salford Systems, 2013. Link: http://www.salford-systems.com

Touw, W. G., Bayjanov, J. R., Overmars, L., Backus, L., Boekhorst, J., Wels, M. \& van Hijum, S. A. (2013). Data mining in the Life Sciences with Random Forest: a walk in the park or lost in the jungle? Briefings in bioinformatics, 14(3), 315-326.

Vuorenkoski, L., Mladovsky, P., \& Mossialos, E.. (2008). Finland: Health system review. Health Systems in Transition, 10(4), 1-168.

Wechsler, D. Wechsler Intelligence Scale for Children. (1949) Psychological Corporation: New York.

Wechsler, D. Wechsler Adult Intelligence Scale. (The Psychological Corporation: New York, 1955).

506 Young, A. F., Powers, J. R. \& Bell, S. L. (2006). Attrition in longitudinal studies: who do you

507 lose? Australian and New Zealand journal of public health, 30(4), 353-361.

508 


\section{Figure 1}

\section{Area of residency}

The area of mothers' place of residency. Dark (red): Helsinki $60 \%$ of mothers (mean distance to hospital $8 \mathrm{~km}$ ). Magenta: Espoo and Vantaa 22\% of mothers (mean distance $15 \mathrm{~km}$ ). Pink: $18 \%$ of the mothers in the surrounding municipalities (average distance $30 \mathrm{~km}$ ). (Adapted from the work of BishkekRocks under CC BY-SA 3.0, http://commons.wikimedia.org/wiki/File:Finnish_municipalities_2007.png) 


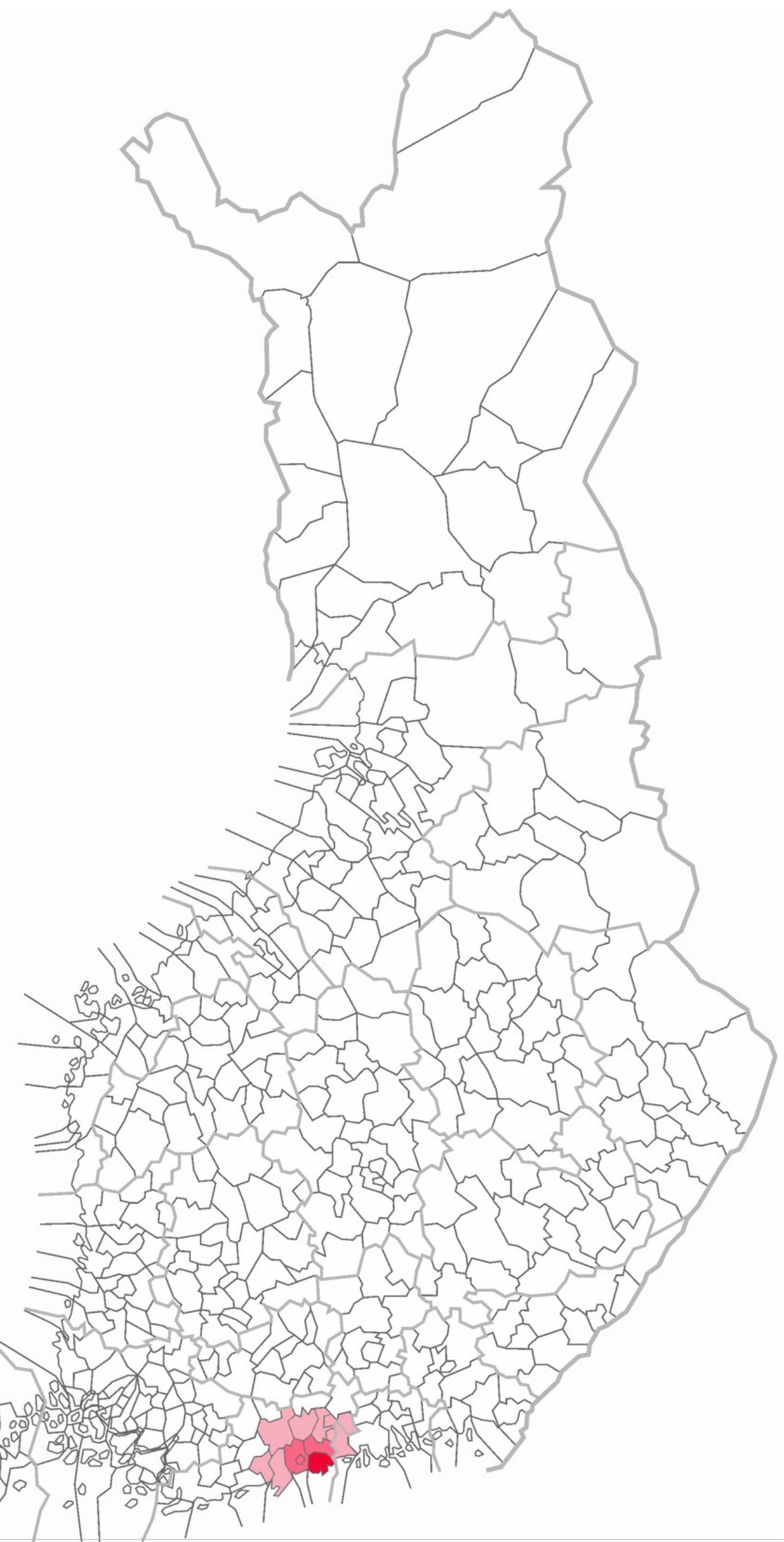

PeerJ reviewing PDF | (v2013:01:225:1:1:NEW 18 Jun 2014) 


\title{
Figure 2
}

\author{
Inclusion
}

Inclusion of subjects in the 30-year longitudinal follow-up study of children at risk for neurodevelopmental disorders.

22359 births $1971-1974$

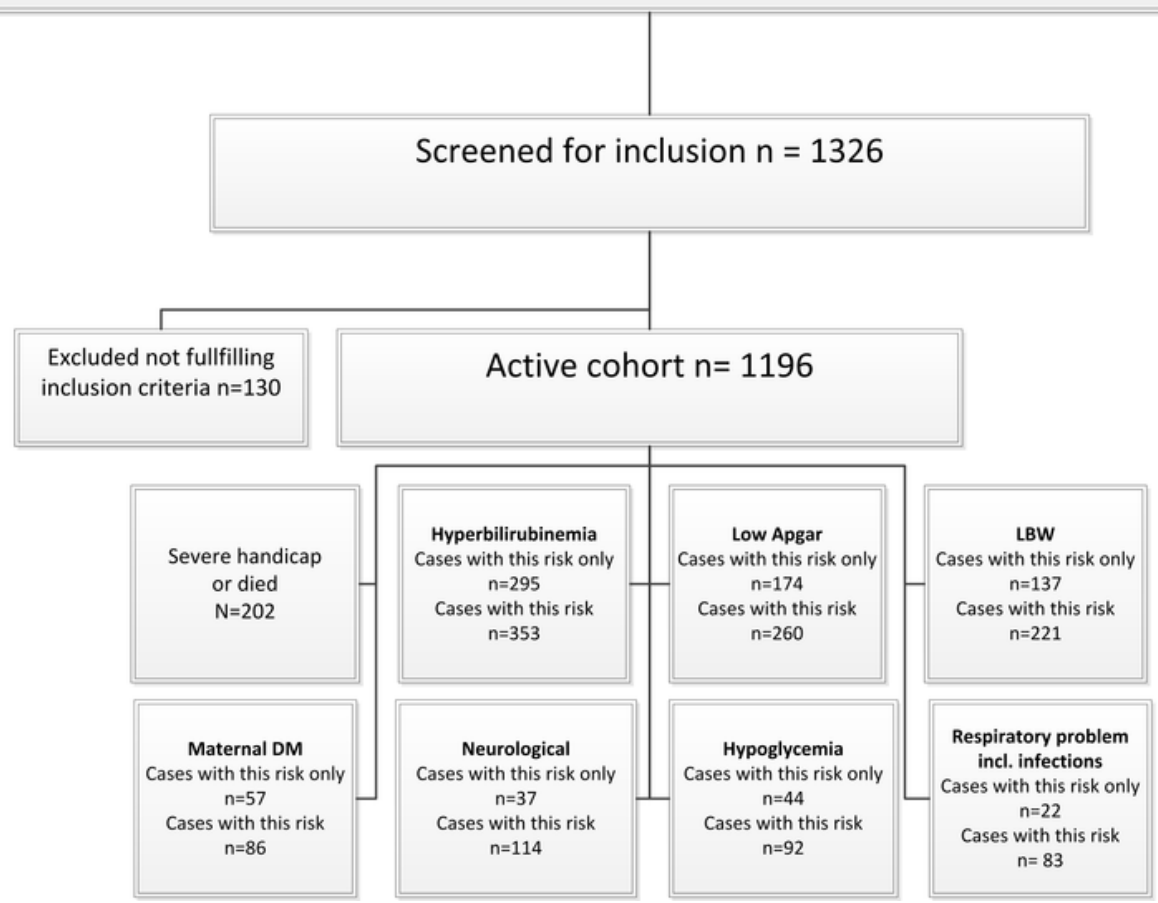

Study inclusion criteria:

- Apgar score $\leq 6$

or $<7$ at 5 or $15 \mathrm{~min}$

- Birth weight $\leq 2000 \mathrm{~g}$ (LBW)

- Bilirubin $\geq 340 \mu \mathrm{mol} / \mathrm{I}$ or blood

exchange transfusion

- Maternal diabetes (DM)

- Hypoglykemia $\leq 1.67 \mathrm{mmol} / \mathrm{l}$

or $\leq 1.21 \mathrm{mmol} / \mathrm{l}$ if premature

- Neurological symptoms

- Severe respiratory problems

- Severe infection 


\section{Figure 3}

\section{Participation}

Participation of subjects in the 30-year longitudinal follow-up study of children in risk of neurodevelopmental disorders. Cases excluded due to death or severe handicap are not shown. Total number of potential participants was 994 at 5 and 9 years, and 864 at 16 and 30 years. Attrition thus was $16 \%$ at five, $24 \%$ at nine, $35 \%$ at 16 and $46 \%$ at 30 years. Numbers on the left column indicate participation at different age levels.

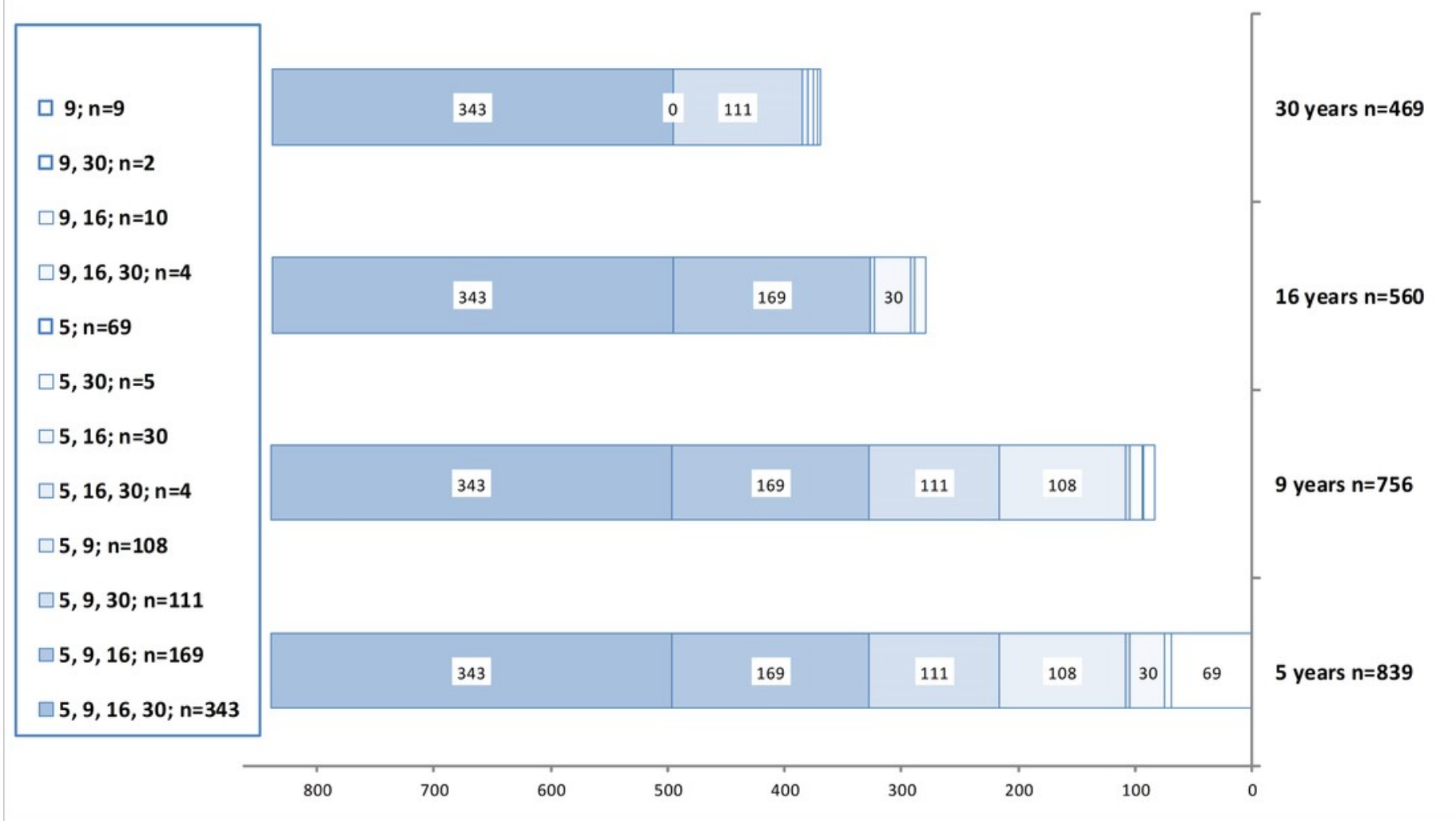




\section{Table $\mathbf{1}_{\text {(on next page) }}$}

Classification accuracy and five of the most important variables

Classification accuracy and five of the most important variables at each age level selected for classification by the random forest classification model. Accuracy (overall fraction correct) calculated from 2 * 2 contingency table $(a+d / t)$. Numbers in parenthesis refer to the age in which the variable is measured. $\mathrm{BMI}=$ body mass index, DAP $=$ Draw a Person test, ITPA = Illinois test of psycholinguistic ability, TOMI = Test of motor impairment, NDS = Neurodevelopmental screen, $\mathrm{WISC}=$ Wechsler intelligence test for children, $\mathrm{VIQ}=$ verbal intelligence quotient, $\mathrm{PIQ}=$ performance intelligence quotient. 


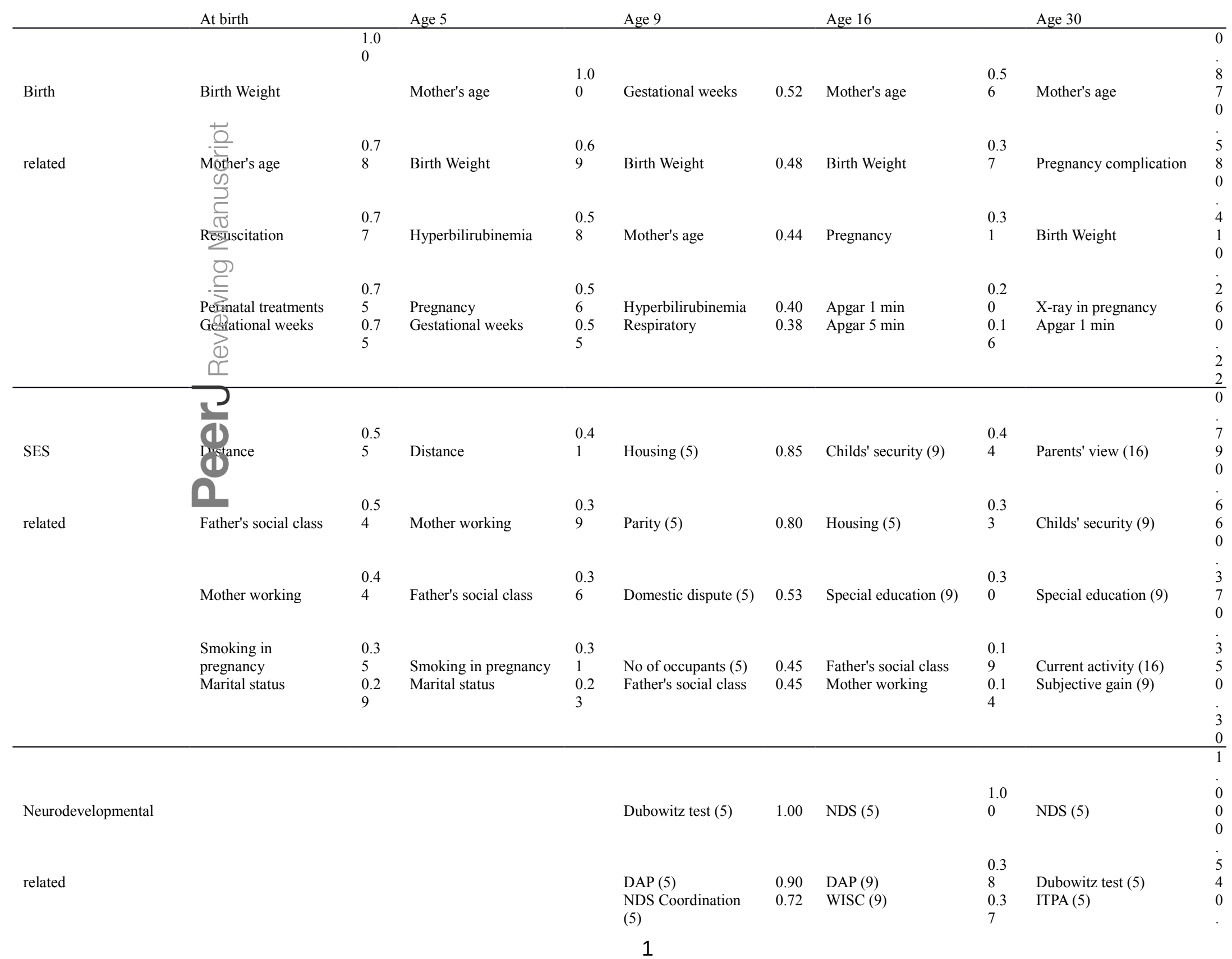




\begin{tabular}{|c|c|c|c|c|c|c|c|}
\hline & & & $\begin{array}{l}\text { ITPA (5) } \\
\text { NDS (5) }\end{array}$ & $\begin{array}{l}0.72 \\
0.71\end{array}$ & $\begin{array}{l}\text { TOMI (9) } \\
\text { ITPA (9) }\end{array}$ & $\begin{array}{l}0.3 \\
3 \\
0.3 \\
2\end{array}$ & $\begin{array}{l}\text { ITPA (9) } \\
\text { WISC VIQ (9) }\end{array}$ \\
\hline & & & & & & & \\
\hline Classification accuracy & $0.8 \%$ & 0.74 & 0.90 & & 0.76 & & 0.96 \\
\hline (95\% confidence limits) & $0.78-0.82$ & $0.71-0.76$ & $0.87-0.91$ & & $0.72-0.79$ & & $0.94-0.97$ \\
\hline
\end{tabular}

Table. Classification accuracy and five of the most important variables at each age level selected for classification by the random forest classification moldel. Accuracy (overall fraction correct) calculated from $2 * 2$ contingency table $(a+d / t)$. Numbers in parenthesis refer to the age in which the variable is measured. BMI = body mass index, DAP = Draw a Person test, ITPA = Illinois test of psycholinguisfíc ability, TOMI = Test of motor impairment, NDS = Neurodevelopmental screen, WISC $=$ Wechsler intelligence test for children, VIQ $\supseteq$ verbal intelligence quotient, PIQ = performance intelligence quotient.

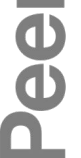




\section{Figure 4}

\section{Retention rate}

The retention rate of the follow-up of a cohort of 1196 neonates with birth risks. The 202 children who died or were severely handicapped were excluded, thus the actual number of included children was 994 (dotted line). ${ }^{*}$ Only the children who had participated at 5 or 9 years were invited at 16 years and 30 years.

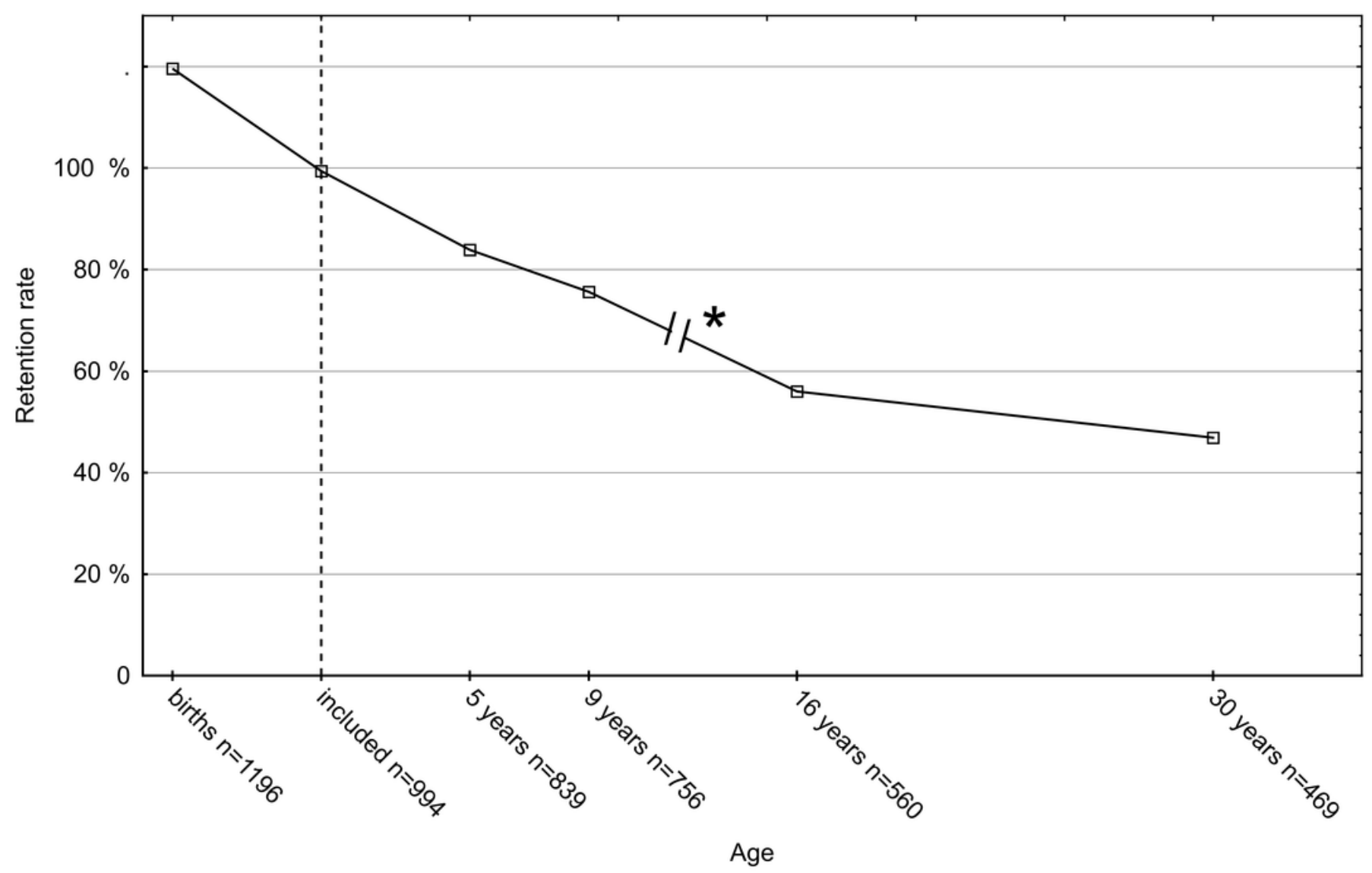




\section{Figure 5}

Variable importance in random forest model

Average importance of variables which predict participation / non-participation as calculated by the random forest classification model. The variables were classified into three categories which are (1) variables relating to child, delivery and pregnancy (Birth), (2) variables relating to neurodevelopment and behavior (Development), and (3) variables reflecting socioeconomic status (SES). Columns represent each age level of the longitudinal follow-up and are scaled to $100 \%$.

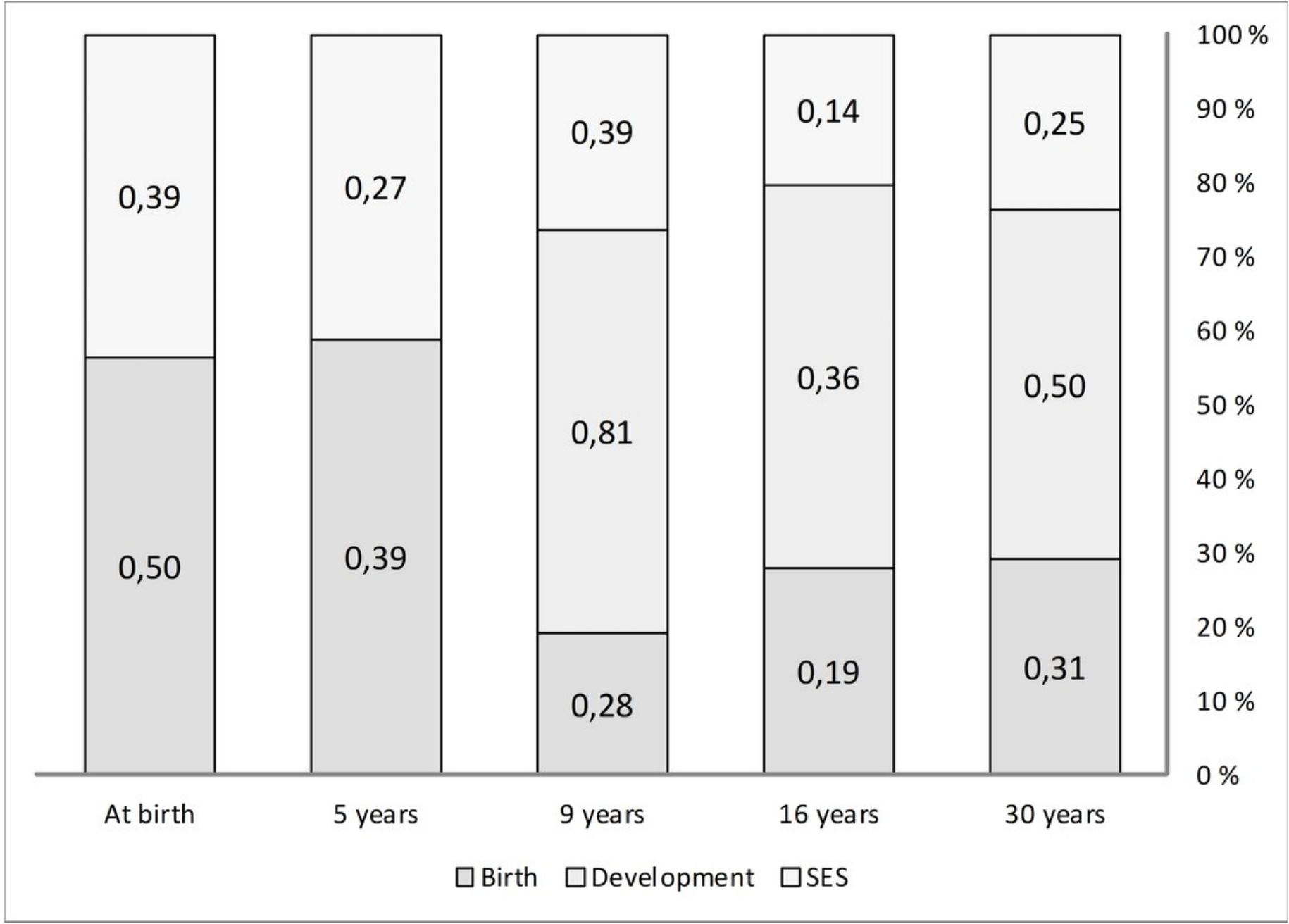

